\title{
A Conversation Between Johnese Spisso and Johannes Czernin
}

\author{
Johnese Spisso ${ }^{1}$ and Johannes Czernin ${ }^{2}$ \\ ${ }^{I}$ University of California at Los Angeles (UCLA), Los Angeles, California; and ${ }^{2}$ David Geffen School of Medicine at UCLA, Los \\ Angeles, California
}

D uring the second week of April, Johannes Czernin, MD, editor-in-chief of The Journal of Nuclear Medicine, talked about COVID-19 response with Johnese Spisso, MPA, President of University of California at Los Angeles (UCLA) Health, CEO of the UCLA Hospital System, and Associate Vice Chancellor of UCLA Health Sciences. She is a nationally recognized academic healthcare leader who oversees all operations of UCLA's hospitals and clinics as well as the health system's regional outreach strategy. Spisso spent 20 years at University of Washington Medicine in Seattle, where she was chief health system officer and vice president of medical affairs for the University of Washington. Trained as a registered nurse, she rose through the ranks at the University of California Davis Medical Center to direct critical care; trauma, burn, and emergency services; and the Life Flight Air-Medical Program. Earlier, she was a critical care nurse in the medical, surgical, and transplant intensive care unit at the University of Pittsburgh Medical Center Presbyterian (PA). Spisso received a master's degree in health-care administration and public administration from the University of San Francisco (CA) and a bachelor's degree in health sciences from Chapman College (Orange, CA). She received her nursing degree at the St. Francis School of Nursing. She has published numerous articles and book chapters on health-care leadership.

Czernin: Johnese, thank you so much for taking the time to talk to our readers. The COVID-19 pandemic has affected every aspect of our lives. Observations from around the world attest to the heroic efforts of first responders, staff, nurses, technologists, administrators, physicians, and researchers. I saw for myself the incredible dedication, kindness, and competence of the healthcare teams in emergency rooms. Can you comment on the level of dedication and commitment of all health-care-related workers worldwide and at your institution?

Spisso: Yes, thank you. You have hit on the most critical element of the response to this pandemic. It is this amazing and extraordinary response that we've had from the entire health-care community in confronting this pandemic. For us here at UCLA Health the response planning actually began in January, when we started to watch some of the events occurring in China. We began working closely with our infectious disease experts and asked about the best initial planning steps. As early as February, we were establishing the elements of our command center so that we could begin to understand how the virus was emerging. But we also assessed what would be needed in personal protective equipment (PPE), in staff training, and how we would quickly develop and create surge capacity. So, it really centered around those 3 things. The critical, essential success factors being our

COPYRIGHT @ 2020 by the Society of Nuclear Medicine and Molecular Imaging. staff, the response of our health-care workers, our PPE to keep them and patients safe. And then the ability to surge up, because, as you know, we were a health system that operated at $100 \%$ capacity. How could we quickly look at changing things so that we could create the additional capacity for COVID-19 patients? We were meeting in our command center every 4 hours.

The formal command center was completely operational 7 days a week by the last week of February. We were having

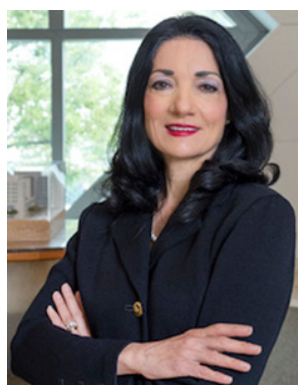

Johnese Spisso, MPA ongoing calls and meetings to assess all the early information. At first, there were concerns about the virus being airborne. We quickly then moved to droplets and appropriate protection. We were making changes on a daily basis so that we could make sure that our staff were protected as we confronted the virus. We also needed to keep all other patients safe. It required an enormous amount of coordination. And really at every level we saw just a tremendous heroic response of staff in wanting to care for patients.

Czernin: You have to take care of large numbers of staff and patients. How many employees does UCLA Health have?

Spisso: We have about 25,000 employees who work in our health system, in our 4 hospitals and in our 180 clinics. It is a very large workforce, and communication has to be precise, concise, and just-in-time so that we can keep people informed. This is especially important in a situation like this, where we have changing information coming forward every day. We really coordinate closely with the Centers for Disease Control (CDC), with the World Health Organization, and with our own Los Angeles County Department of Public Health. This type of communication and disseminating information broadly is really important. Every day we have a 1-hour meeting dedicated just to communications for that next day so that we can proactively align what messages we need to tell staff the next day so that they're prepared for duty.

Czernin: All around the world, healthy citizens and patients fall asleep with the pandemic on their minds, and they wake up with thoughts about this disease. Eventually, this inflicts substantial emotional distress and stress, especially on the entire health-care team. How can you maintain high staff morale for extended periods of time?

Spisso: You're exactly right. And not only are our staff worried about their own safety, they're worried about the safety of their families when they go home after taking care of patients. We have really tried to do a lot of wrap-around services to support staff going the extra mile for us. For example, we provide temporary housing if staff want to stay at hotels rather than going home. 
We're providing additional scrubs, we're providing meals, we're providing assistance with childcare if needed. We also offer our virtual and online wellness programs, mindfulness, meditation, and yoga, as well as reaching out and calling staff, seeing how they're doing, particularly those who are working in our COVID-19 units. Just to really make sure we can continue to support their health and wellbeing and that of their families as they navigate through this crisis and provide the direct frontline care that is so needed.

Czernin: Leadership teams across the world work on emergency preparedness. And in order to be prepared, you have to sacrifice what we would maybe call "nonessential" clinical services. Yet, we also have to serve the patients with other diseases, such as cancer and cardiovascular disease, neurologic disorders, and patients who need surgeries. How can you find a balance between defining something as essential versus nonessential? And how can you make sure that in this time of emergency preparedness, you still have the capability to serve the entire population of diverse patients?

Spisso: That's what's made this pandemic different-because we layered it on top of a very busy health-care system in our country and in the world. For the first time, certainly in my 30year career, we had to take significant dramatic steps to reduce occupancy in other levels of our health-care system. Early in the first week of March, we began reviewing our surgical cases and our procedure cases and postponing anything that wasn't urgent or emergent. There's a term that's used out there, "elective," but in viability of hospital operations is critically important. If I understand you correctly, there needs to be a balance between restarting services for other disease entities and for other procedures as long as they can be done safely and as long as you have the testing capabilities to make sure that this is done safely.

Spisso: Yes, that's our goal. Because, although we were able to safely put off certain procedures for a couple of weeks, we feel that those who have been waiting are definite patient populations that need to have their care resumed.

Czernin: One question about reimbursement. Because the disease is new there cannot be any code for the COVID pandemic or COVID patients. Is this just coded as a pneumonia? How is that done?

Spisso: For example, there are patients who are in the intensive care unit with severe respiratory distress. They will fall into one of those acute respiratory distress syndrome-type categories. Patients who are on the acute care wards with respiratory-compromised pneumonia will fall into those categories. CMS just announced a small enhanced payment for patients coded with COVID-19 reimbursement.

Czernin: That would make the financial impact of the crisis even more profound. Is that correct?

Spisso: Yes, that's correct.

Czernin: Let us think through a hypothetical timeline of this pandemic. It is probably reasonable to assume that this will go on at a certain level for many months. What kinds of actions can a huge health system such as yours take? What are the steps that you

\section{"We were making changes on a daily basis so that we could make sure that our staff was protected as we confronted the virus."}

that elective group there is also a lot of care needed. It needs to be done. And while you may be able to postpone it for a couple of weeks, we're now more than a month into this pandemic. So, we're actually beginning to look at ways to safely restart the health care to the population that we typically serve so that we don't have unintended mortality and morbidity in that population. We now have the ability to do our own COVID-19 testing, and we can test 1,000 patients per day at UCLA Health. We get those results back in a few hours. We therefore feel that we can safely bring some of our patients back who need care by doing the preprocedure testing, making sure that they don't have the virus, or, if they do have the virus and still need the care, we can wear the right PPE. So, it's been the combination of testing and securing enough PPE to really reopen, in a thoughtful way, the health-care system to those patients who need us.

When you look at the month of March, we reduced our surgical and procedure volume by more than $60 \%$ of what we normally do. We also reduced inpatient occupancy by $50 \%$. So right now our hospitals are half occupied. Our surgery and procedure suites are only half occupied. That comes at an enormous expense for the health system as well, because we're not getting the revenues in those cases that help us support all the underserved care that we do. So, we have the expenses of staff who are on paid leave or on benefit time. We have to be thoughtful as this pandemic goes longer than anticipated about how we can still remain financially viable so that we are here to serve the community in the future.

Czernin: The overriding concern here is, of course, about the humane aspect, but, as you mentioned, the issue of financial can take to ramp up in an orderly fashion and make services available for the entire population again?

Spisso: We've been working in 2 ways. First, we are reaching out to our patients who have been postponed and communicating with them on when we'd like to get them back in. We're also sending a weekly letter out to all our patients to update them on the course and development of the pandemic. We offer telemedicine visits with us to check in. The other big aspect has been the work that our UCLA Health Laboratory Medicine has been doing. As you know, we offer our own COVID-19 testing. But we also have another research project that we're doing on plasma from convalescent patients. We identify patients who had the infection and ask them to donate their plasma for therapies. We are working quickly on the antibody testing.

It will be a combination of efforts that will allow us to identify safe caregivers for our patient population. We know that without a vaccine we're going to be dealing with this virus for a while. We could have a reemergence during regular flu season, even though right now we're hopeful that we are starting to see a flattening of that curve.

Our surge was predicted for this past week, and we feel that we're stable. But we have to learn how to continue to provide health care as a health system with the presence of this emerging infectious disease. We, like many other academic health systems, are looking at leveraging our research efforts to get this under control. That is where I think we and the general public are really seeing the value and the power of the academic research that's 
done in many academic facilities around the world. And we're hopeful to secure additional federal funding. We've also had great responses from the philanthropic community that will be able to play a major part in this.

Czernin: Johnese, you mentioned the plasma from convalescent patients. Is that already in clinical trials?

Spisso: We have that information on our uclahealth.org/ coronavirus website, where people can contact our center and donate their plasma for that study.

Czernin: And the antibody testing that you mentioned before, that's supposed to be rolled out fairly soon nationwide?

Spisso: We hope to have that rolled out within the next 2 weeks. We are still working on the protocols for the rollout. Right now, as you know, we're testing all of our preprocedure and presurgical patients for COVID-19. With the antibody testing we hope to be able to test our health-care workers. Antibody testing has now also been made available at UCLA Health.

Czernin: We are all convinced that eventually we will emerge from this crisis. What is your key message for the public and for health-care workers around the world? How can we get through this together?

Spisso: First, I just want to express unbelievable gratitude for our health-care workers, for how they have stepped forward and really rallied to combat this virus, not only here at UCLA, but in our entire state, in the nation, and the world. They really are the heroes behind this. I also would like to thank the general public, particularly in Los Angeles. The response that we have had from the community following the mayor's and the governor's directives in the shelter-in-place and stay-at-home orders-we've really seen the beneficial effects.

We're not in the same situation as some other parts of the country. We can't thank the public enough for their diligence, in that their staying home has allowed us to continue to care for them. I think we have all gotten smarter about transmission of communicable diseases. We will be better and more vigilant as we approach our regular flu season now that more people truly understand how viruses are spread. People are more vigilant about making sure that they are washing their hands, avoiding contact with people who are sick, staying at home when they are sick, covering their sneezes or coughs, cleaning and disinfecting frequently, and following the CDC guidelines. I think that information more than ever is front and center with our public, and we need to keep that up as we go forward.

Czernin: Thank you for taking the time to talk to our readers and to me, and thank you for everything you are doing. 